\title{
An Overview of the Layered and Extensible Aircraft Performance System (LEAPS) Development
}

\author{
Jason R. Welstead* \\ NASA Langley Research Center, Hampton, VA, 23681, United States of America \\ Darrell Caldwell, ${ }^{\dagger}$ Ryan Condotta, ${ }^{\ddagger}$ and Nerissa Monroe ${ }^{\ddagger}$ \\ Analytical Mechanics Associates, Inc., Hampton, VA, 23666, United States of America
}

\begin{abstract}
The Layered and Extensible Aircraft Performance System (LEAPS) is a new sizing and synthesis tool being developed within the Aeronautics Systems Analysis Branch (ASAB) at NASA Langley Research Center. It is a modular, multidisciplinary, multi-fidelity sizing and synthesis tool for modeling advanced aircraft concepts and architectures such as electric/hybrid-electric propulsion, unconventional propulsion airframe integration, and non-traditional mission trajectories. The development of LEAPS is motivated by the lack of existing tools that meet the needs of ASAB. The Flight Optimization System (FLOPS) has been the primary sizing and synthesis tool of ASAB for three decades. However, FLOPS has a number of limitations that make it difficult to use for unconventional aircraft designs. Three high-level goals have been adopted to guide the LEAPS development process. LEAPS is being developed in Python with an architecture built to enable a flexible and extensible analysis capability using the concept of an aircraft object that combines data and analysis models. Five challenge problems for LEAPS have been identified to measure progress: analysis of a conventional tube-and-wing aircraft using legacy methods, coupled aeroelastic analysis for weight estimation of a conventional tube-and-wing aircraft, analysis of an advanced hybrid-electric concept, analysis of the X-57 Maxwell distributed electric propulsion aircraft, and optimization of the trajectory of a supersonic vehicle to minimize sonic boom. LEAPS will be a publicly available capability of exceptional quality with modularity and extensibility that makes it a robust tool for design and analysis of current and future unconventional aircraft concepts.
\end{abstract}

\section{Introduction}

A IRCRAFT design has always been a strongly coupled problem encompassing multiple disciplines, including aerodynamics, structural and component weights, propulsion, and vehicle performance to name a few. The goal has always been to optimize an aircraft for a specified mission (or missions) while trying to minimize, or maximize depending on the design case, a desired objective function.

Traditional analysis methods such as those described in common aircraft design texts ${ }^{1-4}$ work well for conventional transport aircraft configurations when minimizing a common objective function, such as takeoff gross weight (TOGW) or fuel consumption. These methods typically rely on mission segment weight fractions based on historical data to estimate the vehicle TOGW and then use the Breguet range equation to estimate the system level vehicle performance. The Flight Optimization System (FLOPS) ${ }^{5}$ takes this a step further by using a bottoms-up approach to estimate the TOGW combined with a more sophisticated mission analysis capability. FLOPS is an aircraft sizing and synthesis tool continuously developed at NASA Langley Research Center from the early 1980's to 2011. It uses empirical/semi-empirical methods to estimate the vehicle empty weight at the component level, then sums the vehicle empty weight, crew weight, payload weight, and fuel weight to obtain a vehicle gross weight. The mission analysis goes beyond the Breguet range equation and

*Aerospace Engineer, ASAB, NASA Langley Research Center, 1 N Dryden St., M/S 442, Hampton, VA, 23681, Member AIAA.

†Senior Project Engineer, ASAB, NASA Langley Research Center, 1 N Dryden St., M/S 442, Hampton, VA, 23681.

$\ddagger$ Junior Software Developer, ASAB, NASA Langley Research Center, 1 N Dryden St., M/S 442, Hampton, VA, 23681. 
evaluates a complete mission in a series of user-specified mission segments using the energy method first published by Rutowski in $1954 .{ }^{6}$ Each segment can be optimized to a given objective or combination of objectives such as minimum time-to-climb, constant Mach with optimum altitude for specific range, etc.

As demands for improving aircraft environmental performance continue to increase, it will be difficult for tube-and-wing aircraft to meet the aggressive goals of reduced environmental impacts. This is resulting in a deviation from the conventional tube-and-wing configuration to a broad range of potential unconventional concepts for commercial transports of various sizes. Examples of ultra-efficient subsonic transport concepts NASA is exploring with industry include double-bubble lifting fuselage, blended-wing-body, truss-braced wing, and hybrid-wing-body with overwing nacelles. The list of unconventional concepts grows dramatically when electric, hybrid-electric, and turboelectric propulsion architectures are included such as the NASA X-57 Maxwell X-plane.

These concepts present many challenges to legacy sizing and synthesis tools such as FLOPS, but these challenges are not unique to NASA. Development of new tools has become increasingly active across the aircraft conceptual design field as it has been broadly recognized that the current advanced aircraft concepts have deviated sufficiently far from the empirical databases that they can no longer be relied upon to provide accurate predictions for aircraft weights, aerodynamics, propulsion, and performance. The advanced concepts have also become increasingly coupled across multiple disciplines, for example tighter propulsion-airframe integration (PAI) with technologies such as boundary layer ingestion (BLI).

At the NASA Langley Research Center, the Aeronautics Systems Analysis Branch (ASAB) is developing the Layered and Extensible Aircraft Performance System (LEAPS) as the next generation sizing and synthesis tool to replace FLOPS. Formal software development began in Fall 2016, with background research and concept exploration beginning in Fall 2014. Written in Python 3.x, LEAPS is being designed as a highly flexible, modular tool that enables the analysis of not only the current portfolio of advanced aircraft concepts, but also the next generation of advanced concepts on the horizon with as-of-yet unknown technologies. A strong focus is being placed on the tool usability while also providing a means to easily extend the methodology through custom analyses. The user will be able to choose from different levels of fidelity within an analysis model, utilizing low-order analyses where appropriate and selectively utilizing high-order analyses where needed. This paper is focused on providing an overview of LEAPS and describing the long term vision for this new analysis capability.

A detailed discussion of the background and motivation for developing LEAPS, rather than adopting other available tools, is presented in Section II. The goals and objectives that guide the fundamental development of LEAPS are discussed in Section III. The envisioned LEAPS architecture and current development is described in Section IV. Some of the identified challenge problems and required capabilities are discussed in Section V. The paper concludes with a summary of the LEAPS development and some final thoughts in Section VI. This paper is written in conjunction with two companion papers that describe some of the basic low-order methodologies being integrated into LEAPS: weight estimation ${ }^{7}$ and mission analysis, ${ }^{8}$ which builds upon Ref. 9.

\section{Background and Motivation}

Since the mid-1980's the Flight Optimization System (FLOPS) has been the workhorse sizing and synthesis conceptual design tool used for NASA Langley aircraft systems analysis studies. A monolithic FORTRAN code, the tool was continuously enhanced by the lead developer, Arnie McCullers, through version 8.2, which was released for government use in 2011. Since this time, the tool has been nearly stagnant with the exception of minor bug fixes. In 2016, a publicly available version was released and denoted FLOPS 9.0. The greatest strength of FLOPS has been its extreme versatility; nearly every component of the analysis can be tuned, giving users the ability to explore the design space, validate a model to known data, and perform sensitivity and trade studies. This capability has been the greatest contributor to the longevity of FLOPS, making it a tool used throughout industry, academia, and government organizations. Additionally, the same tool was capable of analyzing subsonic tube-and-wing transports, blended-wing-body subsonic transports, supersonic transports, and fighter aircraft.

Unfortunately, FLOPS has reached the end of its operational capability given the previously described advanced concepts that have risen to prominence in recent years. Advances in electric propulsion, both as a power source and electrical system architecture, have been increasingly difficult to model using the FLOPS software. Recent analyses have been successful through the use of integration frameworks, such 
as ModelCenter and OpenMDAO, where FLOPS has been manipulated to perform the required analyses. Examples include setting the engine thrust specific fuel consumption (TSFC) to nearly zero to simulate a battery-powered aircraft, performing various analyses outside of FLOPS and applying the applicable overrides, and using outdated data columns in the FLOPS output in conjunction with a convergence loop in the integrated analysis framework to achieve a desired analysis. Although these workarounds were sufficient at the time, it has reached the point where more manipulations than analyses are performed in order for FLOPS to generate the desired output.

The greatest limitations of FLOPS lie within the fundamental assumptions that are applied to the analyses. The fundamental assumptions include: 1) fuel is consumed during the mission, 2) the aerodynamic characteristics are only a function of Mach number, altitude, and lift coefficient, 3) the vehicle is a point mass with a single degree of freedom (energy altitude), and 4) only a single propulsion type (fuel or electric) can be used during any mission segment. The mission analysis is no longer capable of capturing the active design constraints and system sensitivities that are shaping the advanced concept design space being explored today. The FLOPS aerodynamic analysis insufficiently captures the propulsion-airframe coupling utilized by recent advanced concepts where the vehicle aerodynamic performance is a function of propulsion system performance (throttle setting). Current state-of-the-art commercial transport aircraft fall outside the FLOPS empirical weight database causing the weight estimation routine to inaccurately capture trends and sensitivities. These internal analyses required significant enhancement to improve the modeling of unconventional configurations. It is because of these limitations that a requirement for a new mission analysis and synthesis capability has been identified.

To address the shortcomings of these legacy aircraft design tools, an internal NASA team was established to chart a path forward to bridge gaps in analysis required for robust synthesis of unconventional aircraft configurations, including those with electric/hybrid-electric propulsion system architectures. Instead of immediately embarking on a new tool development, a survey of developed conceptual design and analysis tools was conducted to understand the established and new state-of-the-art approaches. With the aid of a NASA-funded study at the Georgia Institute of Technology, twenty-two conceptual design tools and software suites were identified while acknowledging that there exist even more unidentified. Although the aircraft conceptual design community is relatively small, every organization appears to have their own preferred analysis tools and methods. The identified tools included: Environmental Design Space (EDS) ${ }^{10}$ Aircraft Performance Program (APP) ${ }^{\mathrm{a}}$, Advanced Aircraft Analysis (AAA) ${ }^{\mathrm{b}}$, Project Interactive ANalysis and Optimization (Piano) ${ }^{\mathrm{c}}$, Pacelab Aircraft Preliminary Design (PacelabAPD) ${ }^{11}$ Aircraft Design Software (ADS) ${ }^{\mathrm{d}}$, FLight OPtimization System (FLOPS), ${ }_{5}^{5}$ AirCraft Synthesis (ACS) ${ }^{\mathrm{e}}$, Transport Aircraft System OPTimization (TASOPT) ${ }^{12}$ Program for Aircraft Synthesis Studies (PASS), ${ }^{13}$ Stanford University Aerospace Vehicle Environment (SUAVE), ${ }^{14-18}$ Preliminary Aircraft Design and Optimization (PrADO) ${ }^{19}$ Airliner COnceptual DEsign (ACODE), ${ }^{20}$ Boeing Mission Analysis Program (BMAP) ${ }^{21}$ Truss Braced Wing Multidisciplinary Optimization Design Environment, ${ }^{22}$ Aircraft Engine Design System Analysis Software (AEDsys) ${ }^{\mathrm{f}}$, RDSg, Nicolai's Sizing Program ${ }^{\mathrm{h}}$, VAMPzero, ${ }^{23} \mathrm{MachUp}^{\mathrm{i}}$, Propulsion Airframe iNTegration for Hybrid Electric Research (PANTHER), Aircraft Preliminary Sizing Tool (PreSTo) ${ }^{j}$, and the Computational Research and Engineering for Acquisition Tools and Environments - Air Vehicles project (CREATE-AV) DaVinci. ${ }^{24-28}$ Many of the identified conceptual design tools rely on regression analyses and/or historical correlations to incrementally improve upon previous designs for similar aircraft configurations and missions. Several tools consist of proprietary methods and are not easily modified or enhanced. Tools such as BMAP and DaVinci are not available due to being commercial proprietary or limited government release, neither of which fosters community collaboration.

SUAVE and TASOPT were explored in detail due to their recent development and broad availability. SUAVE is a Python-based open-source environment that enables multi-disciplinary and multi-fidelity analysis of advanced concepts. It is both a framework and a mission and performance analysis tool that is highly

\footnotetext{
attp: //www.darcorp.com/Software/app/

${ }^{b}$ http: //www. darcorp.com/Software/AAA/

${ }^{\mathrm{c}}$ http://www.piano.aero/

${ }^{\mathrm{d}}$ http://www.oad.aero/

e http://www.avidaerospace.com/software/avid-acs/

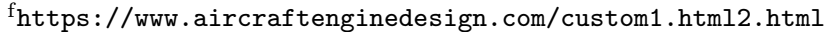

gttp://www. aircraftdesign.com/rdspro.html

h http://www.dept.aoe.vt.edu/ mason/Mason_f/MRsoft.html\#Nicolai

i https://www.blucraft.com/machup/

jhttp://www.fzt.haw-hamburg.de/pers/Scholz/PreSTo.html
} 
flexible, designed to analyze a broad range of missions and vehicle architectures. Recently demonstrated in Ref. (16) was the coupling of OpenVSP, Gmsh, and SU2, an open-source flow solver developed at Stanford University, for the analysis of a conventional tube-and-wing transport, a blended-wing-body transport, and a supersonic transport. The framework was used to generate a surrogate aerodynamic model for use in mission analysis that is informed by high-order aerodynamic analysis.

A complex mission can easily be set up in SUAVE as a series of segments, but segments such as climb and descent cannot be easily optimized for minimum time or fuel, a common practice in conceptual design mission modeling. Additionally, the rate-of-climb, velocity, and altitude must be specified. However, it is often useful to optimize the top-of-climb altitude to maximize the cruise performance, subject to an excess power constraint on the propulsion system. The SUAVE architecture enables the analysis of both conventional and unconventional vehicles through its analysis flexibility, and the user is able to modify the source code as required without having to recompile. However, leveraging this capability requires the user to have a strong familiarity with the source code and the interactions of the various components and libraries. Another limitation is the lack of sufficient documentation (at the time of evaluation) and the large number of variables and classes that must be appropriately connected to obtain meaningful results. Finally, the computational expense of evaluating a mission that requires an iterative solution, such as determining the aircraft TOGW for a specific mission, is excessively high. This becomes a limiting factor when performing a large optimization, trade study, or design of experiments (DoE).

Transport Aircraft System Optimization (TASOPT) is an analysis tool developed at the Massachusetts Institute of Technology that couples the analysis and optimization of an aircraft with the analysis and optimization of a turbine engine. ${ }^{12}$ This provides a closely coupled airframe and engine design and analysis, leading to both being fully optimized simultaneously rather than two suboptimal designs stitched together. TASOPT is especially strong in the evaluation of concepts that include boundary layer ingestion, a technology that relies upon synergistic propulsion-airframe integration to achieve a system level performance benefit. TASOPT uses fundamental physics-based methodologies for much of the vehicle analysis while trying to minimize the use of correlation and empirical-based relations.

A drawback of TASOPT is that the tool was designed to always optimize the vehicle for a specified mission. This creates issues when performing an independent assessment of a concept that has already been sized. The allowable mission definition lacks flexibility, which proves problematic for electric/hybridelectric concepts. TASOPT was written as a monolithic FORTRAN code, which means that the tool has the benefits of the improved execution time of a compiled code, but it is challenging modify or completely replace analyses. This reduced flexibility limits the tool's capability to analyze a broad range of vehicle types and architectures without significant modification similar to the ASAB tool, FLOPS. The input file requires detailed information not always available early in the conceptual design process, such as component material properties and temperature limits. There is an extensive theory manual for TASOPT which helps the user understand the methodologies implemented in TASOPT, but the lack of a user's manual makes it extremely difficult to create an input file from scratch or deviate significantly from an example input file.

Each tool was used to model the Central Reference Aircraft data System (CeRAS), ${ }^{29}$ a conventional tubeand-wing aircraft model based on the A320 used to baseline modeling and analysis tools. After evaluating both TASOPT and SUAVE, it was determined that the best solution for ASAB was to develop a new, in-house aircraft conceptual design software. One option considered was to leverage the currently available software as a starting point, but it was not desired to have multiple versions of TASOPT or SUAVE. There also existed within ASAB a strong desire to have complete control over the software development as opposed to contributing to a non-NASA controlled open source development such as SUAVE.

The motivation for developing LEAPS is to first and foremost meet the needs of our primary customer, NASA. These needs include being able to design and evaluate a broad range of aircraft concepts and propulsion system architectures. The high degree of coupling between airframe and propulsion technologies in future advanced concepts requires new, innovative analysis techniques that are suitable for conceptual design but can be informed by higher fidelity analyses. Although the focus is the design of advanced aircraft concepts, the capability to independently evaluate external concepts or as-built aircraft is absolutely required for ASAB to act as an independent broker for NASA decision makers. FLOPS excelled at performing an independent analysis, while allowing the user to "tweak" the underlying analyses through the flexible use of input variables to account for various technology assumptions. Moving forward, it is desired to retain this capability while adding enhanced flexibility for a modular analysis tool that enables multidisciplinary and multi-fidelity analysis. 
It is also desired to reach out in a collaborative manner to address some of the challenges of non-NASA customers such as the Department of Defense or academia. With the ever changing landscape of advanced concepts and technologies, active development of new analysis methods and techniques will ensure a continued capability for analyzing the state-of-the-art. Any new analysis methods developed as part of LEAPS should be shared with the aircraft design community. This collaborative environment is highly desired as part of the NASA push to release internally developed software to the public domain. To foster this collaboration, LEAPS will be released as Publicly Available including all source code and user documentation.

Even though LEAPS is being developed as a completely new software, this does not imply that legacy methods are obsolete or externally developed methods cannot be integrated. The desire is to leverage analysis techniques and methodologies as much as feasible without compromising the ability to publicly release. Particular emphasis is being placed on developing an operational tool, not just a research tool, that provides an exceptional user experience and robust analysis with informed feedback. LEAPS should be usable by all aircraft designers and not just a limited set of researchers that actively develop the code.

In summary, the motivations for developing LEAPS are 1) a lack of tools that meet internal customer needs for the analysis of advanced aircraft concepts and propulsion architectures using multidisciplinary and multi-fidelity analyses, 2) a desire to collaborate on aircraft concepts and analysis methods in the public domain, 3) the lack of software with robust error checking, informative exception messages, and complete user and theory documentation, and 4) the inability to easily modify, enhance, or extend analyses as new technologies and computational capabilities are developed.

\section{Goals and Objectives}

A set of high-level goals were established to provide a unified vision for the LEAPS tool development. These goals provide the overarching guidelines for establishing development objectives for the software architecture, analysis models, and tool execution. The high-level goals are:

1. develop a modular, multidisciplinary, multi-fidelity aircraft design and performance software tool;

2. create an aircraft design and analysis tool of exceptional quality and performance; and

3. enable the ease of distribution to the aeronautical community.

The first goal is quite simple in its description; design an analysis tool capable of performing the breadth of analyses required by NASA systems analysts using a range of analysis fidelities. LEAPS is not an effort to drive high-order analysis tools to all aspects of aircraft conceptual and preliminary design. Instead, the focus of LEAPS is to enable the right analysis for the right job at the right time. This thinking allows the user to choose which analysis fidelity to use while understanding the trade between computational time and analysis order. This goal drives the development to enable a flexible analysis capability for the next generation of sizing and synthesis of advanced aircraft concepts. The objectives that support the first high-level goal are:

- support analysis of a broad range of aircraft, including: transports to fighters, tube-and-wing to blended wing body, and jet fuel to all electric propulsion;

- incorporate an energy agnostic framework allowing the analysis of a broad range of traditional and future power systems, energy storage systems, and energy harvesting systems;

- support multi-order, fully-coupled analysis and simulation built centrally around a mission performance core; and

- be capable of providing the relevant information to support the latest tools for optimization, sensitivity analysis, and design of experiments.

The second high-level goal places an emphasis on delivering a high quality software. Often, analysis tools are developed in direct response to a research question that requires a timely answer. As such, an analysis tool is hastily developed that answers the research question but is highly restricted in its capability and usability; usually, only the tool developer is able to glean usable answers from the software. This is reactionary tool development and is often necessary to meet organizational research demands. LEAPS is

being developed as a proactive tool development. An analysis capability gap has been identified, but no specific research question is being answered. As a proactive tool development, special emphasis has been 
placed on utilizing standard software development practices, such as enforced version control, code reviews, unit testing, and simultaneous documentation and development, to deliver a software of exceptional quality. This approach is enabled by having a team of both exceptional aerospace engineers and software engineers working closely together.

A positive user experience drives the architecture development to ensure that the software is both capable and useful. As capability grows, the usability can decrease or be subject to an extreme learning curve for new users. Through careful user-interface design, the goal is to provide a highly capable software while decreasing the learning curve for new users. A major aspect of this is informative feedback from the tool, such as clear exception messages that provide direction to resolution, status updates, detailed feedback upon request (verbose), and clear output data that is easy to interpret and clearly labeled (with units). When successfully implemented, LEAPS will be a high-quality tool with significant capability and a positive user experience.

As was mentioned previously, there is a trade between analysis fidelity and computational cost. The user ultimately makes this trade in their analysis setup. However, there is also a trade between computational speed and ease of development when choosing a programming language. A strength of FLOPS is its ability to perform a complex analysis - including optimizing mission trajectories - in approximately one second. If the FLOPS methodology was identically implemented in Python, the analysis time would be measurably longer. No emphasis has been placed on hitting a target analysis time, but instead an emphasis has been placed on having sufficient computational performance such that the user can obtain usable information in a timely manner to answer his/her research questions. The objectives that summarize the second high-level goal are:

- develop LEAPS using standard software engineering development practices;

- be of a modular design with a flexible architecture; and

- provide an exceptional user experience in terms of stability, reliability, and informed exception messages.

The final high-level goal for LEAPS focuses on the strong desire to foster collaboration within the aircraft conceptual design community. The NASA open source initiative has placed an emphasis on distributing tools and capabilities developed through tax-payer dollars to the research community. LEAPS will not be released as open source. Instead, LEAPS will be released as publicly available, which is only subtly different, to enable collaboration on methods, analysis techniques, and results. The supporting objective for this final goal is clear and concise: develop LEAPS with procedures and practices that enable easy distribution of methods and software to the aeronautics community.

\section{Software Architecture}

The LEAPS software architecture was designed to achieve the high-level goals and objectives described in Section III. In addition to the goals and objectives, experiences solicited from a broad range of FLOPS users have shaped the LEAPS architecture design.

\section{A. User Stories}

In order to maximize the quality of the user experience, multiple FLOPS users were interviewed to gain their insights and experiences. These user stories directly influenced the LEAPS architecture design and development. Those interviewed included newer users, users with moderate experience, and experts in order to obtain a broad range of feedback and experiences, all of which could be captured in the LEAPS development.

In these user stories certain key features were consistently mentioned. Well-liked FLOPS features were suggested for LEAPS, one being the short computation time to run a single analysis case. FLOPS can execute a complete analysis in approximately one second. LEAPS will be designed so that execution time is sufficiently fast with specific care given to profile the code and eliminate unnecessarily excessive computation time. Another positive FLOPS feature identified was the assumed default values for input variables. Having good default values for most user inputs enables a rather complicated FLOPS analysis with a very short input file. The user can increase the fidelity of the input and the analysis as they gain more information 
about the design, but they do not have to jump from a zero solution state to a full solution state. An extensive override capability allows users to modify internally computed values with either an absolute value or scalar multiplier. This is useful when modifying an internal analysis to match data produced by an external analysis, such as a finite element model.

Also, the highly formatted text output, including the input echo, was viewed very positively by most users. This enables the easy use of differencing tools, parsing the output for feeding other analysis or frameworks, and being able to easily use the features of advanced text editors.

The major complaint of FLOPS users centers on the lack of exceptions and warnings. FLOPS in many ways does not exit gracefully and thus provides the user little to no insight as to why the execution failed. If convergence loops do not complete, there is little or no warning. Also, it was found that there is a lack of robust and clear error handling. Errors and warnings are intermixed in the output, which is of sizable length, and they can be easily missed when reviewing the output. Numerous other minor issues were identified, but for conciseness, only these major themes that were influential in the LEAPS software architecture have been highlighted.

\section{B. Architecture Design}

Leveraging the FLOPS user experiences, the LEAPS architecture was designed to present four general user interfaces: command line, input file, output stream, and log files. The command line allows the user to request help, specific modes and/or analyses, or optional data. The command line also provides the capability to both specify an input file and, for the purpose of capturing the output stream, a output file path. The input file allows the user to request analyses and specify related data and/or notes for requested analyses. It allows the user to logically separate data according to configurations, aircraft components, etc., for analysis. The output stream provides the user with the results of requested analyses as text. By default, LEAPS generates a $\log$ file that provides the user with regular status updates and any requested optional data. This information allows the user to determine if LEAPS is performing as expected. These user interfaces run under a simple concept of execution, which is divided into the four phases of startup, setup, update, and report. The startup and setup phases initialize variables and do preliminary calculations. The update phase performs the bulk of the calculations. The report phase outputs the results of the calculations. The flow of the concept of execution is shown in Fig. 1.

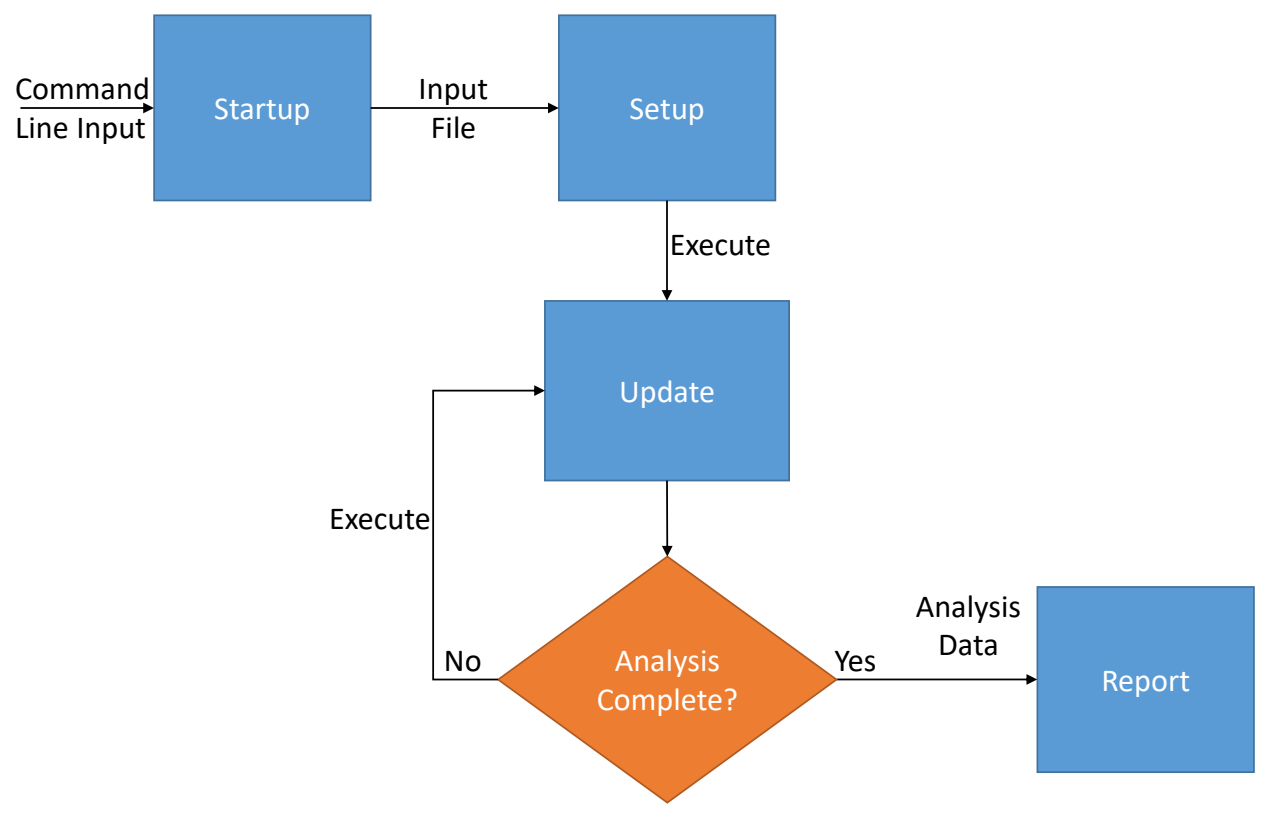

Figure 1. LEAPS concept of execution.

LEAPS data exchange is based on the concept of an "aircraft" object and the user-specified analysis models. An "aircraft" object is composed of modeled physical behaviors and supporting data sets logically grouped together in components. The model requested by the user dictates the analyses and required input 
and derived data. LEAPS supports at a minimum five model concepts per aircraft:

- The energy model calculates thrust production and power consumption. Thrust and power are included here to model propulsion systems of all types. Aircraft can use mass exchange, internal combustion, electrical power, or some combination of one or more propulsion types, to produce thrust. Power for other systems may be a byproduct of thrust production. Additional energy storage may be available and/or required for those same systems or propulsion.

- The weights model calculates the dynamic weights of the aircraft and its subsystems as a function of the mission. It also calculates the static (constant with time) weights of an aircraft and its subsystems.

- The atmosphere model defines density, temperature, and speed of sound as a function of altitude. The default atmosphere model is for Earth. Simulating flight conditions on Earth using a different fidelity model or on another planet can be achieved by specifying an alternative atmosphere model that is provided by the user.

- The aerodynamics model calculates lift and drag properties of an aircraft based on thrust, weight, an atmosphere model, and the current geometric configuration. Additional aerodynamic properties such as moments can be calculated as required via higher analysis fidelity when using a more complex mission model.

- The mission model manages the different mission segments from takeoff through landing. Analyses can focus on only one segment, or can focus on the following combinations: takeoff and flight, flight and landing, or takeoff, flight, and landing. Flight is defined as a series of segments (climb, cruise, descent, turn, loiter, etc.) for lower level analysis and a full flight trajectory simulation for higher level analysis. Maneuvers may be included as part of the flight phase of a mission.

Each model consists of a set of layers, as shown in Fig. 2, that represent various analysis levels dependent upon user input. Higher Levels are associated with increasing analysis order and complexity within a model. Higher analysis Levels require increased user input and input fidelity. Each model represents a customization point. End users are permitted to use and/or write replacement models as desired using a Model Package Interface. Each model package shall provide its own set of model specific handlers. Handlers add components to an "aircraft" object by reading input data streams, and they write "aircraft" object components to output data streams. The handlers are instantiated and invoked as needed through a common package interface, as such handlers are an internal implementation detail. This gives each model package maintainer the option to implement appropriate handlers as needed.

LEAPS' extensible nature allows the user to run

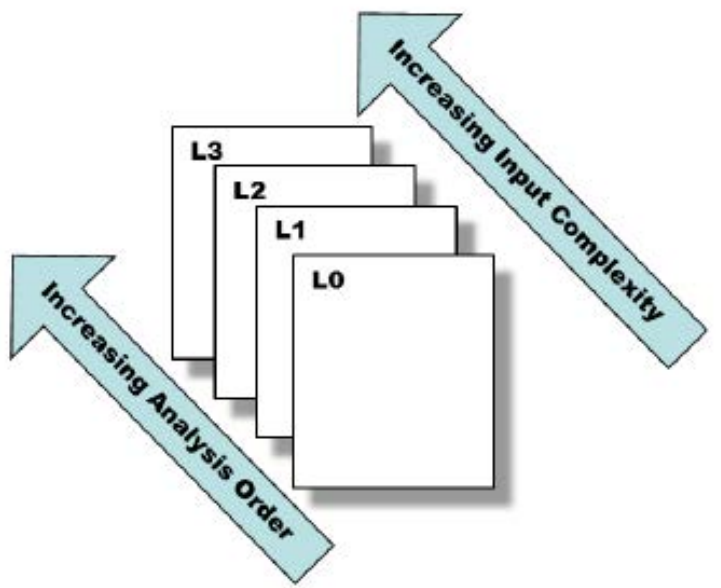

Figure 2. Various analysis levels of LEAPS models. analyses in a multitude of ways. The user can customize LEAPS by adding new models and/or removing default models for an analysis. When creating new models, client code will use the LEAPS Model Package Interface, which is a set of required functions implemented in a client model or package. From this interface, the user has the ability to replace any of the models with their own implementation.

LEAPS can also be externally driven by another program. This means that LEAPS, whether it be a single model such as weights or the entire LEAPS core, can be plugged into another external driver and executed. The LEAPS core, as represented in Fig. 3, is a combination of the input interface, the output interface, and the models of LEAPS. These are the core components necessary to run LEAPS. The ability to be externally driven enables the user to pull the key components, or the entirety, of LEAPS that they are interested in and perform numerical techniques and operations such as optimization. The separation of numerical techniques such as optimization from LEAPS is to enable the user to run their desired techniques 
and not limit them to only a LEAPS provided capability. For this to occur, the interface between the external driver and LEAPS must be created. The combination of customizing and selecting models provides the user with a capability to use aspects of LEAPS they find useful, while not limiting them to features provided. The multitude of interfaces, as shown in Fig. 3, create an extensible and flexible tool.

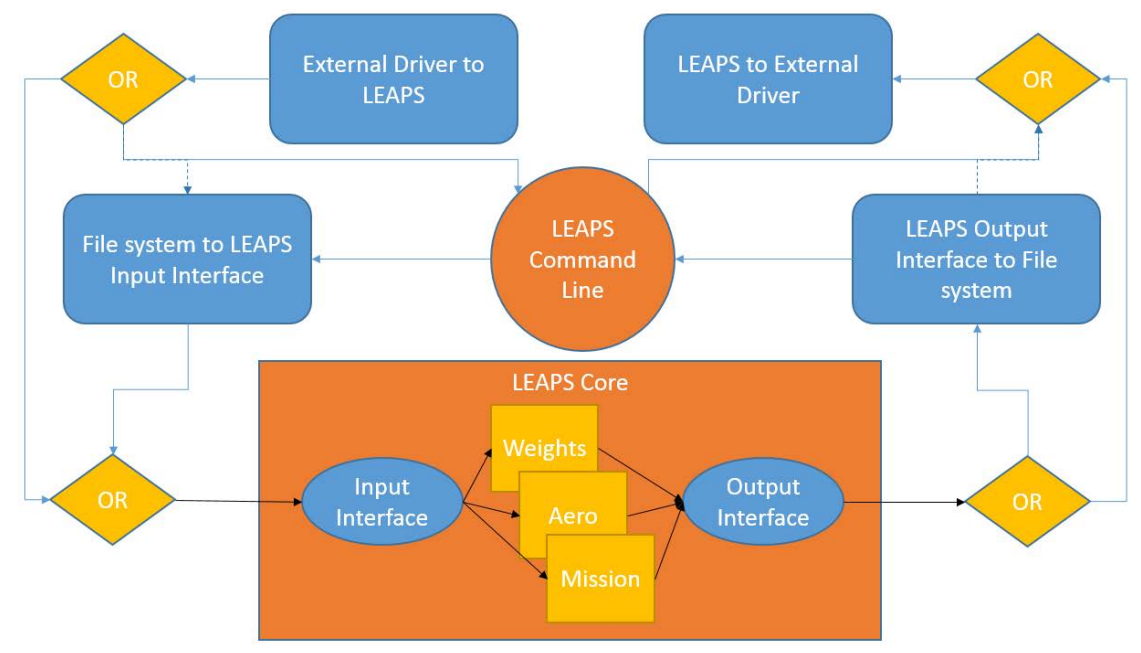

Figure 3. LEAPS input/output interfaces.

\section{LEAPS Input File Structure}

The LEAPS input file uses a configuration file format (.ini). This file format was selected over other formats such as XML, CSV, and JSON. The Python package library provides support for INI files that is straightforward and concise, and the file structure and organization is simple and easy to understand. This file is separated into comments, sections, and properties. The comments are used for denoting noninitialization information and are ignored by the LEAPS parser. The sections and the properties capture initialization data. In Fig. 4 a comparison of the FLOPS input file and LEAPS input file is shown. A few differences indicated are the structure, names, and notes aspects. As shown in Fig. 4, the input file is organized efficiently according to component structure and verbose terminology. It even allows the notes to be organized according to the variable with which they are associated. These advantages allow the user to quickly organize an input file and run an analysis with ease.

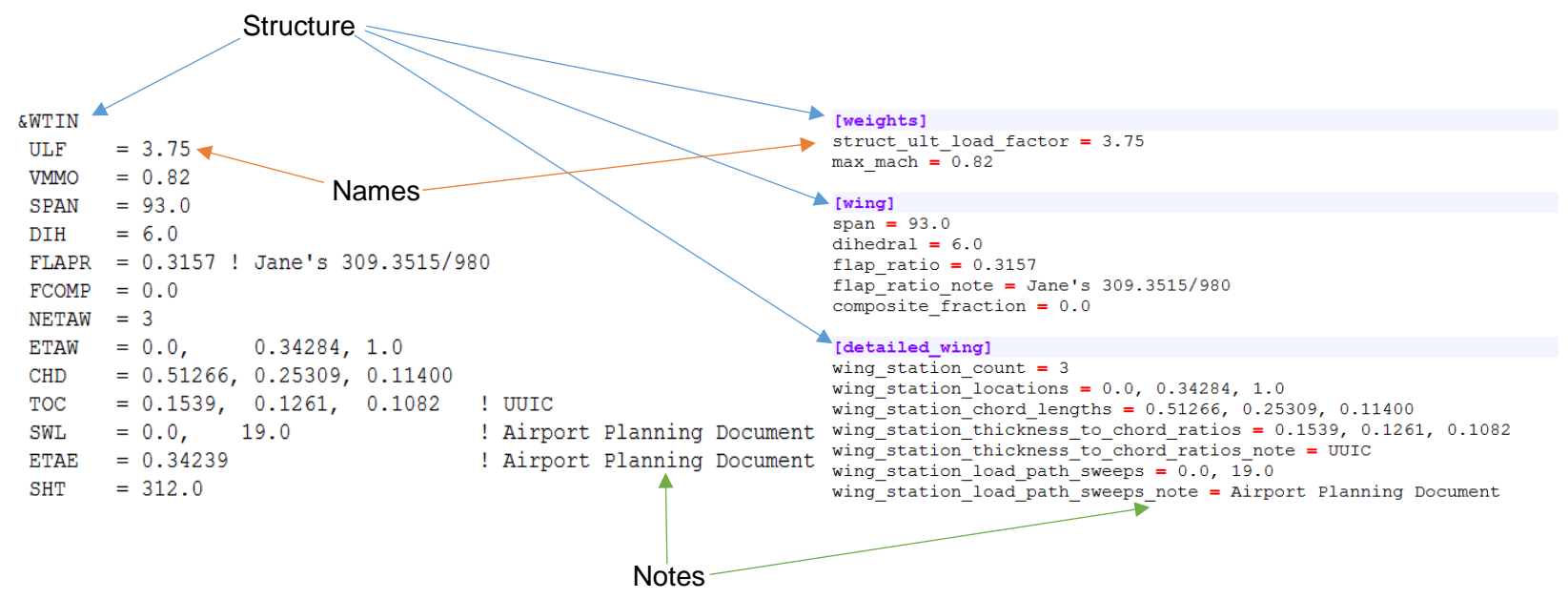

Figure 4. Comparison of the legacy FLOPS input file with a namelist-based organization and the new LEAPS input file using the initialization file format. 


\section{Description of Identified Challenge Problems}

Any new tool that replaces a legacy tool must first show that it can perform the analysis of the legacy tool. In this case, LEAPS is replacing FLOPS which excelled at accurately modeling tube-and-wing aircraft when compared to published data. LEAPS must be able to replicate this capability since many advanced concepts are compared to an equal technology conventional tube-and-wing baseline aircraft. The analysis methods used in the evaluation of conventional aircraft still retain validity, and so these legacy methods are being utilized as much as possible in LEAPS and expanded as required. A set of challenge problems have been identified to guide the LEAPS development, where each problem requires enhanced capability to be solved. When LEAPS is capable of handling the challenge problems, it will have significant capability far beyond the current ASAB legacy tools.

The first challenge problem is the analysis of a conventional tube-and-wing aircraft that leverages legacy methods and combines them with the new LEAPS architecture and mission performance analysis (see Ref. 8). These results will be directly compared to a FLOPS analysis as there is high confidence in the quality of the FLOPS analysis in this case. The goal is not to replicate the results of FLOPS - there should be minor disagreements due to changes in methodology — but to instead show that the analysis results are consistent between the tools and the differences are clearly understood. This first challenge problem is focused on verification and validation of the LEAPS methods and architecture while obtaining feedback on the user experience.

The second challenge problem builds upon the first challenge problem by incorporating a coupled aeroelastic analysis on a tube-and-wing configuration. Technology improvements in both aerodynamics and structures are enabling aircraft to improve vehicle level performance by increasing wing span and aspect ratio. For span constrained aircraft, such as single-aisle aircraft complying with Class C airport operations, the span is fixed, but the aspect ratio continues to incrementally increase. The higher wing aspect ratios and spans have sufficiently deviated from the legacy database such that empirical wing weight estimates are not capturing the appropriate trends. Cantilevered wings are transitioning from being stress constrained to aeroelastic constrained (tip deflection and flutter). This challenge problem will leverage an extension of LEAPS for a coupled aero-structural analysis to improve the structural weight estimates of the primary structure. The challenge lies in sufficiently capturing the static and dynamic aeroelastic constraints without compromising the ability to perform an entire analysis in a timely manner.

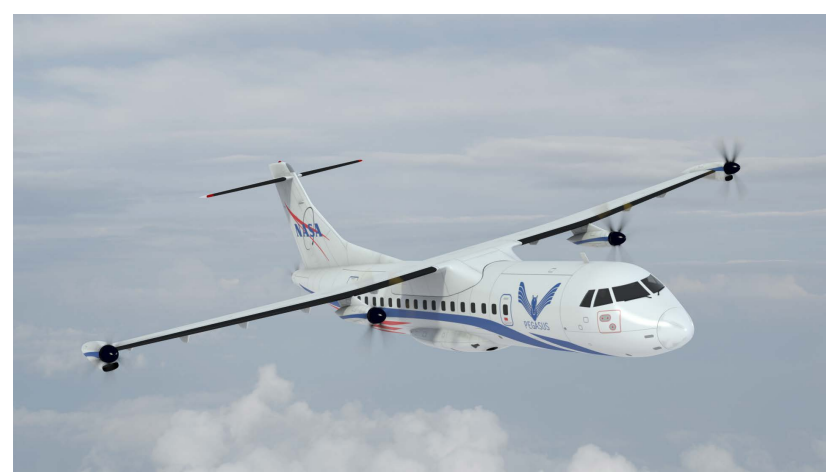

Figure 5. Artistic rendering of the PEGASUS concept. ${ }^{30}$
The third challenge problem is the design and analysis of the advanced aircraft concept PEGASUS (Parallel Electric-Gas Architecture with Synergistic Utilization Scheme). ${ }^{30}$ The PEGASUS concept, shown in Fig. 5, is a regional aircraft designed specifically to revitalize the short-haul market by leveraging hybrid-electric technologies and synergistic PAI to minimize the vehicle operating cost. Multiple propulsors are used on this concept including inboard electric propulsors, turbine-electric wingtip propulsors, and an unducted tailcone BLI propulsor. This concept utilizes multiple unique propulsors, an electric/hybrid-electric architecture that for short missions can operate on batteries alone, and flexibility in the split of electric energy versus fuel energy. Analysis of this concept requires significant capability enhancement beyond legacy tools to account for multiple propulsor models with individual throttling control throughout the mission, tracking of energy instead of fuel to converge on total energy usage, and robust PAI modeling. LEAPS must also be driven by an external driver to optimize the electric split of the aircraft architecture.

The fourth identified challenge problem is the analysis of the X-57 Maxwell aircraft. The X-57 is an allelectric manned demonstrator with distributed propulsors along the wing leading edge and cruise propellers on the wingtips, as is shown in Fig. 6(a). Analyzing this aircraft will exercise the new energy agnostic architecture for calculating the all-electric mission performance, but a greater challenge lies in capturing the highly throttle dependent aerodynamic effects. The success of the X-57 concept lies in its ability to capitalize on the distributed propeller aero-propulsive coupling, that in the simplest description, acts like a 

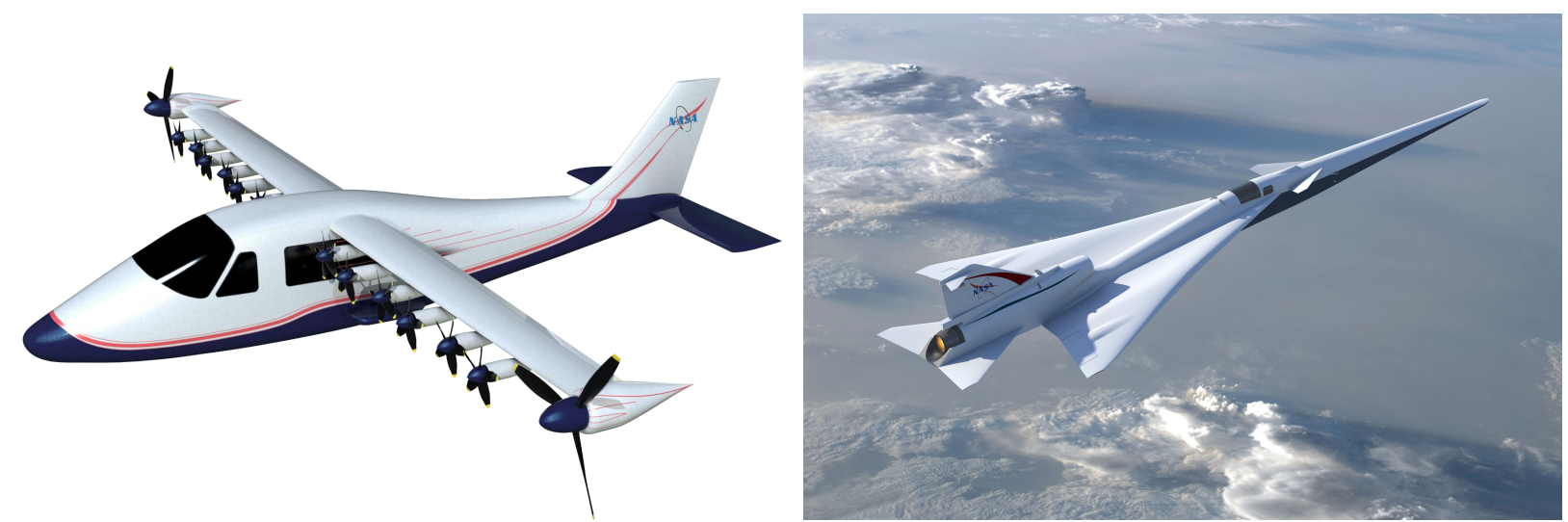

(a) X-57 Maxwell electric aircraft with leading edge distributed (b) Rendering of the Quiet Supersonic Transport (QueSST). ${ }^{\mathrm{k}}$ propulsion. ${ }^{31,32}$

Figure 6. NASA X-plane concepts identified as LEAPS challenge problems.

high-lift system. Additional complexity is added to the architecture by also leveraging synergistic PAI with the wingtip propellers. The expectation is that flight test data from the demonstrator will be available to aid in performing this analysis.

The final identified challenge problem is to perform a mission analysis for a supersonic vehicle in which the trajectory has been optimized to minimize a boom propagation signal. To perform this analysis a series of CFD solutions will need to be generated such that a surrogate model can be used for the offbody pressure profiles. This is assuming that it is infeasible to compute off-body pressure profiles during analysis execution due to prohibitively long execution time, but in reality that could be done with enough computational resources and patience. LEAPS will have to be extended to include the surrogate model, a sonic boom propagation tool such as $\mathrm{sBOOM}^{33}$ and a user custom objective function for the mission segment or segments being optimized with respect to the boom signature. An example configuration to use for this challenge problem is the Quiet Supersonic Transport (QueSST) shown in Fig. 6(b).

\section{Summary}

The Layered and Extensible Aircraft Performance Systems (LEAPS) is being developed as the next generation aircraft sizing and synthesis tool for the Aeronautics Systems Analysis Branch (ASAB) at NASA Langley Research Center, replacing the legacy Flight Optimization System (FLOPS) software. This tool is being proactively developed to address the current and future challenges in the modeling of advanced aircraft concepts and technologies. The development is being accomplished through a modular, multidisciplinary, multi-fidelity analysis capability with an architecture intentionally designed to be flexible and extensible. The motivation for developing this new tool was to meet the needs of ASAB, close the current analysis capability gaps, foster collaboration in methods development and the analysis of advanced concepts, and develop a software that provides a positive user experience and is capable of being enhanced for years to come. These motivations for developing LEAPS were mapped directly into the high-level software development goals and objectives. The software architecture design was described in detail in Section IV and has been developed to achieve the high-level goals. Finally, some long term challenge problems have been identified to provide development direction for continued capability enhancement.

\section{Acknowledgments}

This research was funded by both the Transformational Tools and Technologies (TTT) Project, part of the Transformative Aeronautics Concepts Program (TACP), and the Advanced Air Transport Technologies (AATT) Project, part of the Advanced Air Vehicles Program (AAVP) within the NASA Aeronautics Research Mission Directorate (ARMD).

\footnotetext{
${ }_{\mathrm{k}}$ https ://www.nasa.gov/press-release/nasa-completes-milestone-toward-quieter-supersonic-x-plane
} 


\section{References}

${ }^{1}$ Raymer, D. P., Aircraft Design: A Conceptual Approach, 3rd ed., American Institute of Aeronautics and Astronautics, Reston, VA, 2006.

${ }^{2}$ Nicolai, L. M. and Carichner, G. E., Fundamentals of Aircraft and Airship Design: Volume I, AIAA Education Series, American Institute of Aeronautics and Astronautics, Reston, VA, 2010.

${ }^{3}$ Roskam, J., Airplane Design, Pt. II, DARcorporation, Ottawa, KS, 1997.

${ }^{4}$ Torenbeek, E., Synthesis of Subsonic Airplane Design, Delft University Press, Delft, Holland, 1982.

${ }^{5}$ McCullers, L., "Aircraft Configuration Optimization Including Optimized Flight Profiles," Proceedings of the Symposium on Recent Experiences in Multidisciplinary Analysis and Optimization, No. NASA CP2327, 1984, pp. 395-412.

${ }^{6}$ Rutowski, E. S., "Energy Approach to the General Aircraft Performance Problem," Journal of the Aeronautical Sciences, Vol. 21, No. 3, 1954, pp. 187-195.

${ }^{7}$ Horvath, B. L. and Wells, D. P., "Aircraft Conceptual Design Weight Estimation Comparison to the Flight Optimization System Method," AIAA Aerospace Sciences Meeting, American Institute of Aeronautics and Astronautics, 2018, (To Be Published).

${ }^{8}$ Capristan, F. M. and Welstead, J. R., "An Energy-Based Low-Order Approach for Mission Analysis of Air Vehicles in LEAPS," AIAA Aerospace Sciences Meeting, American Institute of Aeronautics and Astronautics, 2018, (To Be Published).

${ }^{9}$ Capristan, F. M. and Welstead, J. R., "LEAPS: An Initial Assessment Towards a Multi-Order Approach to Air Vehicle Mission Analysis," 18th AIAA/ISSMO Multidisciplinary Analysis and Optimization Conference, American Institute of Aeronautics and Astronautics, Denver, CO, 2017, pp. 1-16, AIAA-2017-4325.

${ }^{10}$ Kirby, M. R. and Mavris, D. N., "The Environmental Design Space," 26th International Congress of the Aeronautical Sciences, 2008, pp. 1-9.

${ }^{11}$ Locatelli, D., Riggins, B., Schetz, J. A., Kapania, R. K., Robic, B., Leenaert, C., and Poquet, T., "Aircraft Conceptual Design: Tools Evaluation," 14th AIAA Aviation Technology, Integration, and Operations Conference, American Institue of Aeronautics and Astronautics, Atlanta, GA, June 2014, pp. 1 - 38, AIAA-2014-2030.

${ }^{12}$ Greitzer, E. M., et al., "N+3 Aircraft Concept Designs and Trade Studies, Final Report," Contractor Report Volume II, National Aeronautics and Space Administration, 2010, NASA/CR-2010-216794.

${ }^{13}$ Antoine, N. E., Aircraft Optimization for Minimal Environmental Impact, Ph.D. thesis, Stanford University, August 2004.

${ }^{14}$ Wendorff, A. D., Botero, E., and Alonso, J. J., "Comparing Different Off-the-Shelf Optimizers' Performance in Conceptual Aircraft Design," 17th AIAA/ISSMO Multidisciplinary Analysis and Optimization Conference, American Institute of Aeronautics and Astronautics, Washington, D.C., June 2016, pp. 1-14, AIAA-2016-3362.

${ }^{15}$ Variyar, A., Economon, T. D., and Alonso, J. J., "Multifidelity Conceptual Design and Optimization of Strut-Braced Wing Aircraft using Physics-Based Methods," 54th AIAA Aerospace Sciences Meeting, American Institue of Aeronautics and Astronautics, San Diego, CA, January 2016, pp. 1 - 13, AIAA2016-2000.

${ }^{16}$ MacDonald, T., Botero, E., Vegh, J. M., Variyar, A., Alonso, J. J., Orra, T. H., and Ilario da Silva, C. R., "SUAVE: An Open-Source Environment Enabling Unconventional Vehicle Designs through Higher Fidelity," 55th AIAA Aerospace Sciences Meeting, American Institute of Aeronautics and Astronautics, Grapevine, TX, January 2017, pp. 1-17, AIAA-2017-0234. 
${ }^{17}$ Botero, E., Wendorff, A. D., MacDonald, T., Variyar, A., Vegh, J. M., Lukaczyk, T., Alonso, J. J., Orra, T. H., and Ilario da Silva, C. R., "SUAVE: An Open-Source Environment for Conceptual Vehicle Design and Optimization," 54th AIAA Aerospace Sciences Meeting, American Institute of Aeronautics and Astronautics, San Diego, CA, January 2016, pp. 1-17, AIAA-2016-1275.

${ }^{18}$ Lukaczyk, T., Wendorff, A. D., Botero, E., MacDonald, T., Momose, T., Variyar, A., Vegh, J. M., Colonno, M., Economon, T. D., Alonso, J. J., Orra, T. H., and Ilario da Silva, C., "SUAVE: An OpenSource Environment for Multi-Fidelity Conceptual Vehicle Design," 16th AIAA/ISSMO Multidisciplinary Analysis and Optimization Conference, American Institue of Aeronautics and Astronautics, Dallas, TX, June 2015, pp. 1 - 56, AIAA-2015-3087.

${ }^{19}$ Strohmeyer, D. and Seubert, R., "Improvement of a Preliminary Design and Optimization Program for the Evaluation of Future Aircraft Projects," rth AIAA/USAF/NASA/ISSMO Symposium on Multidisciplinary Analysis and Optimization, American Institute of Aeronautics and Astronautics, 1998, pp. 1-8, AIAA1998-4828.

${ }^{20}$ Lefebvre, T., Schmollgruber, P., Blondeau, C., and Carrier, G., "Aircraft Conceptual Design in a MultiLevel, Multi-Fidelity, Multi-Disciplinary Optimization Process," 28th International Congress of the Aeronautical Sciences, International Congress of the Aeronautical Sciences, 2012, pp. 1-11.

${ }^{21}$ Bradley, M. K. and Droney, C. K., "Subsonic Ultra Green Aircraft Research: Phase I Final Report," Contractor Report NASA/CR-2011-216847, NASA Langley Research Center, Hampton, VA, April 2011.

${ }^{22}$ Gur, O., Bhatia, M., Mason, W. H., Schetz, J. A., Kapania, R. K., and Nam, T., "Development of Framework for Truss-Braced Wing Conceptual MDO," 51st AIAA/ASME/ASCE/AHS/ASC Structures, Structural Dynamics, and Materials Conference, American Institute of Aeronautics and Astronautics, 2010, pp. 1-32, AIAA-2010-2754.

${ }^{23}$ Böhnke, D., Nagel, B., and Gollnick, V., "An Approach to Multi-fidelity in Conceptual Aircraft Design in Distributed Design Environments," 2011 IEEE Aerospace Conference, 2011, pp. 1-10.

${ }^{24}$ Livingston, J. W., "Aeronautical System Design and Physics-Based Simulation," 55th AIAA Aerospace Sciences Meeting, American Institute of Aeronautics and Astronautics, Grapevine, TX, 2017, pp. 1-11, AIAA-2017-1195.

${ }^{25}$ Roth, G. L., Livingston, J. W., Blair, M., and Kolonay, R., "CREATE-AV DaVinic: Computationally Based Engineering for Conceptual Design," 48th AIAA Aerospace Sciences Meeting, American Institute of Aeronautics and Astronautics, Orlando, FL, 2010, pp. 1-17, AIAA-2010-1232.

${ }^{26}$ Brooks, G. P., Livingston, J. W., Adamec, S., and McGough, W., "HPCMP CREATE-AV DaVinci 3.0 and HPCMP CREATE-MG Capstone Integration," 53rd AIAA Aerospace Sciences Meeting, American Institute of Aeronautics and Astronautics, Kissimmee, FL, 2015, pp. 1-17, AIAA-2015-1508.

${ }^{27}$ Rooney, B. D., "The CREATE-DaVinci Power User Experience," 52nd Aerospace Sciences Meeting, American Institute of Aeronautics and Astronautics, National Harbor, MD, 2014, pp. 1-7, AIAA-2014-0921.

${ }^{28}$ Post, D. E., Atwood, C. A., Newmeyer, K. P., Meakin, R. L., Vogelson, R. L., Hariharan, N., Morton, S., Livingston, J., D’Angelo, J. N., Dey, S., Gorski, J., Moyer, E. T., Mackenna, A., and Strawn, R., "The CREATE Program: Design and Analysis Tools for DoD Weapon Systems," 54th AIAA Aerospace Sciences Meeting, American Institute of Aeronautics and Astronautics, San Diego, CA, 2016, pp. 1-17, AIAA-2016-0562.

${ }^{29}$ Risse, K., Schäfer, K., Schültke, F., and Stumpf, E., "Central Reference Aircraft data System (CeRAS) for research community," CEAS Aeronautical Journal, Vol. 7, No. 1, 2016, pp. 121-133.

${ }^{30}$ Antcliff, K. R. and Capristan, F. M., "Conceptual Design of the Parallel Electric-Gas Architecture with Synergistic Utilization Scheme (PEGASUS) Concept," AIAA/ISSMO Multidisciplinary Analysis and Optimization Conference, American Institute of Aeronautics and Astronautics, Denver, CO, June 2017, pp. 1 - 15, AIAA-2017-4001. 
${ }^{31}$ Borer, N. K., Patterson, M. D., Viken, J. K., Moore, M. D., Clarke, S., Redifer, M. E., Christie, R. J., Stoll, A. M., Dubois, A., Bevirt, J., Gibson, A. R., Foster, T. J., and Osterkamp, P. G., "Design and Performance of the NASA SCEPTOR Distributed Electric Propulsion Flight Demonstrator," 16th AIAA Aviation Technology, Integration, and Operations Conference, American Institute of Aeronautics and Astronautics, Washington, D.C., 2016, pp. 1-20, AIAA-2016-3920.

${ }^{32}$ Dubois, A., van der Geest, M., Bevirt, J., Clarke, S., Christie, R. J., and Borer, N. K., "Design of an Electric Propulsion System for SCEPTOR's Outboard Nacelle," 16th AIAA Aviation Technology, Integration, and Operations Conference, American Institute of Aeronautics and Astronautics, Washington, D.C., 2016, pp. 1-30, AIAA-2016-3925.

${ }^{33}$ Rallabhandi, S. K., "Advanced Sonic Boom Prediction Using the Augmented Burgers Equation," Journal of Aircraft, Vol. 48, No. 4, 2011, pp. 1245-1253. 